\title{
MMSE Limitations for Subband Adaptive Equalisers
}

\author{
Hafizal Mohamad ${ }^{1}$, Stephan Weiss ${ }^{1}$, and Markus Rupp ${ }^{2}$ \\ ${ }^{1}$ Dept. Electronics \& Computer Science, University of Southampton, UK \\ ${ }^{2}$ Institute of Communications and RF Engineering, TU Wien, Vienna, Austria \\ \{hm99r,sw1\}@ecs.soton.ac.uk, mrupp@nt.tuwien.ac.at
}

\begin{abstract}
In this contribution, we investigate limitations of the minimum mean square error (MMSE) solution for subband adaptive equalisers due to aliasing and error in power complementarity. By deriving the Wiener solution for the subband equaliser and comparing it against simulations, we verify that aliasing caused in the subband decimation can be considered equivalent to channel noise. We discuss how these limitations can be mitigated by careful system design.
\end{abstract}

\section{Introduction}

Linear distortion in a communication channel caused by multi-path propagation and limited bandwidth leads to inter-symbol interference (ISI) at the receiver, which in turn can result in a potentially considerable bit error probability in the detection [1]. Fig. 1 shows the arrangement of a channel $c[n]$ as well as an equaliser $w[n]$ that is used to compensate for the distortion introduced by the channel. Adaptive equalisers have been widely employed to mitigate the effect of ISI and to retrieve a transmitted signal $u[n]$ from a distorted received copy $x[n]$ at the output of a channel $c[n]$ in the presence of channel noise $v[n]$. For minimum mean square error (MMSE) equaliser, adaptive algorithms can be employed to adjust the equaliser $w[n]$ by minimising the error between the received signal $y[n]$ and a known training sequence in a suitable sense.

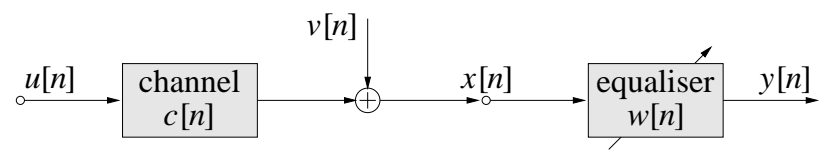

Fig. 1. Block diagram with channel impulse response $c[n]$, channel noise $v[n]$, and equaliser $w[n]$.
Adaptive equalisers based on subband decomposition methods have recently been demonstrated to exhibit faster convergence speed at a lower computational complexity than their fullband counterparts when long equaliser responses $w[n]$ are adapted by least mean square (LMS) algorithms [2, 3]. In related adaptive filtering applications, subband techniques have previously been proposed for echo cancellation applications $[4,5]$ where the achievable MMSE performance has a lower limit imposed by the employed filter bank structure through aliasing in the subbands [6]. In this paper we will investigate if similar limitations exist and can be quantified for subband adaptive equalisers.

The paper is organised as follows. A review of subband equalisation and oversampled modulated filter banks (OSFBs) to perform the decomposition into subbands is presented in Sec. 2. The quality of OSFBs is characterised by the alias level in the subbands, and the power complementarity (PC) of the filter bank system. In Sec. 3 we hypothesise that aliasing has a channel-noise-like regularisation effect on the subband MMSE solution and subsequently derive the Wiener solution for subband equalisers. In Sec. 4 we compare this Wiener solution, based on aliased and unaliased system components with simulation results, validating our hypothesis. Sec. 5 investigates the influence of PC on the MMSE solution. Sec. 6 discusses the impact of this finding in the context of equaliser performance as well as its design. Finally, conclusions are drawn in Sec. 7.

\section{Subband Equaliser Structure}

The schematic of a subband adaptive equaliser is shown in Fig. 2. At the receiving end of the communications system, the subband equaliser is trying to compensate for the channel distortion by minimising the error $e[n]$ between the received signal $x[n]$ and the training sequence $d[n]$ which is delayed version of the transmitted data $u[n]$. In the subband system, both signals $x[n]$ and $d[n]$ are split into $K$ subband signals decimated 


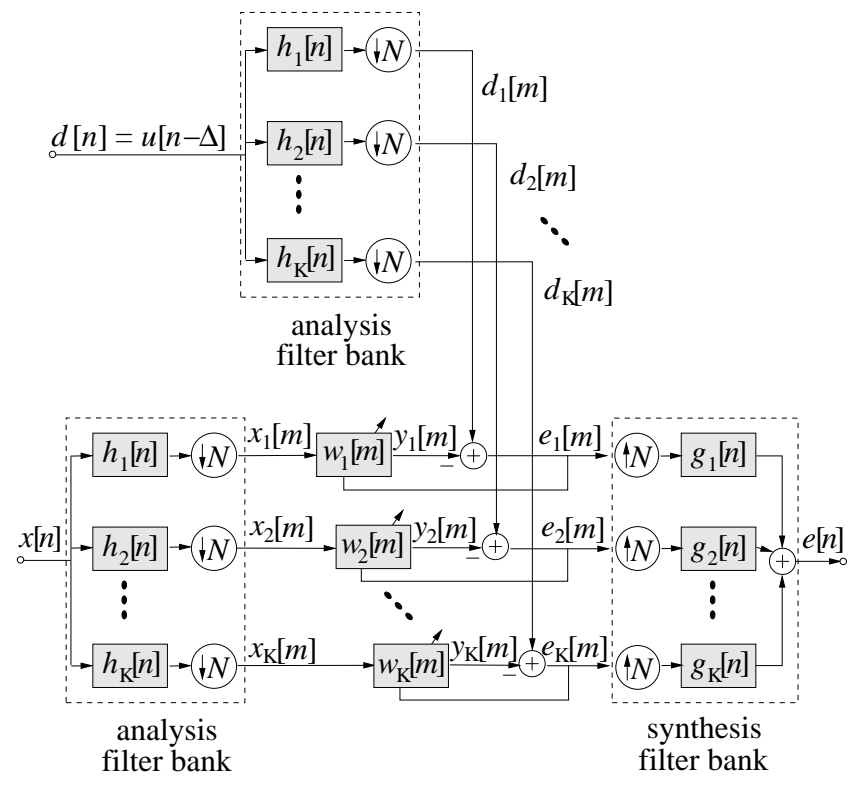

Fig. 2. Subband adaptive equaliser structure.

by a factor of $N$ by analysis filter banks. The resulting subband signals $x_{k}[m]$ and $d_{k}[m]$ are used to adapt the equaliser $w_{k}[m]$ independently in each corresponding $k$ th subband. By using an appropriate adaptation algorithm, such as the LMS, the subband error $e_{k}[m]$ can be minimised. Finally, a fullband output signal $y[n]$ can be reconstructed from the $y_{k}[m], k=1(1) K$, by means of a synthesis filter bank. In Fig. 2, instead of $y[n]$, the error signal $e[n]$ is reconstructed in order to assess the fullband performance of the subband equaliser.

Oversampled modulated filter banks with a decimation of $N<K$ are used for subband adaptive equalisation such that aliasing is restricted to the stopband of the analysis filters and can therefore be controlled by appropriate filter bank design [6]. Analysis filters $h_{k}[n]$ and synthesis filters $g_{k}[n]$ are derived from a real-valued lowpass prototype FIR filter $p[n]$ by using a generalised discrete Fourier transform (GDFT, [7]). An example of the characteristic of such an analysis filter bank is given in Fig. 3.

In general there are two main criteria that should be taken into consideration in designing the filter banks. First, aliasing occurs as the filter bank output in Fig. 2 is decimated. Second, subband systems should have perfect reconstruction (PR) or near PR property such that the filter banks impose no or only a negligible distortion on the overall system. The PR condition refers to a concatenation of an analysis and a synthesis filter bank resembling a perfect delay [8]. If the alias level in the various subbands is low enough, PR is fulfilled

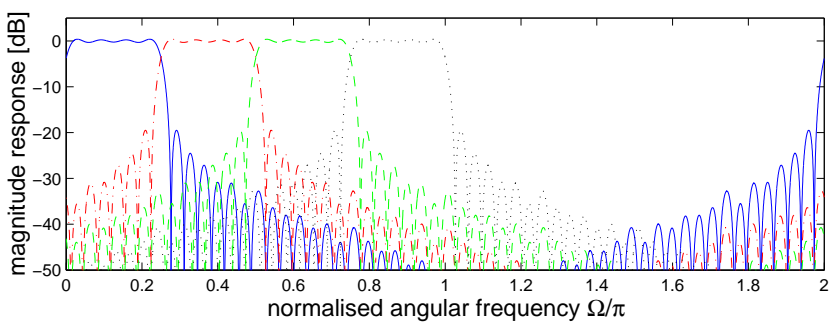

Fig. 3. Magnitude response and arrangement of bandpass filters for $K=8$ and $N=7$ for GDFT modulated filter banks, only showing half the bands covering the spectrum $[0 ; \pi]$.

if the filter bank system is PC, i.e. the filter characteristics such as shown in Fig. 3 yield a flat overall spectrum. Consequently, the finite stopband attenuation and inaccuracies of the filter banks are likely to introduce limitations for the MMSE solution of a subband equaliser, which will be further explored in the following sections.

\section{Wiener Solution}

In this section, the Wiener solutions for fullband and subband equalisers will be presented where the latter is derived in order to investigate the effect of aliasing on the optimum MMSE solution of the subband system. For conventional fullband equaliser, it is known that the equaliser's ability to combat ISI is limited by the channel noise $v[n]$ [1]. In reference to Fig. 1, applying the Wiener estimation of the transmitted signal $u[n]$ based on the noisy measurement $x[n]$, the optimal MMSE solution to $w[n]$ is given by the Wiener filter in the frequency domain as [9]

$$
W_{\text {opt }}\left(e^{j \Omega}\right)=\frac{P_{u u}\left(e^{j \Omega}\right) \cdot C^{*}\left(e^{j \Omega}\right)}{P_{u u}\left(e^{j \Omega}\right) \cdot\left|C\left(e^{j \Omega}\right)\right|^{2}+P_{v v}\left(e^{j \Omega}\right)} .
$$

This assumes that $u[n]$ and $v[n]$ are statistically independent. In our notation, $P_{u u}\left(e^{j \Omega}\right)$ and $P_{v v}\left(e^{j \Omega}\right)$ are the power spectral densities (PSDs) of $u[n]$ and $v[n]$, respectively, and $c[n] \circ-C\left(e^{j \Omega}\right)$ is the Fourier transform of the channel impulse response. Note that the fullband MMSE equaliser performance is influenced through the regularisation term in (1) by the signal to noise ratio (SNR) of the transmission system [10].

For the subband equaliser, we establish the hypothesis that aliased signal components can be treated as channel noise. In the $k$ th subband, we adopt the model depicted in Fig. 4. The sequences $u[n]$ and $v[n]$ correspond to the outputs of source models $f_{1, k}[n]$ and $f_{2, k}[n]$ which are excited by white Gaussian signal of 


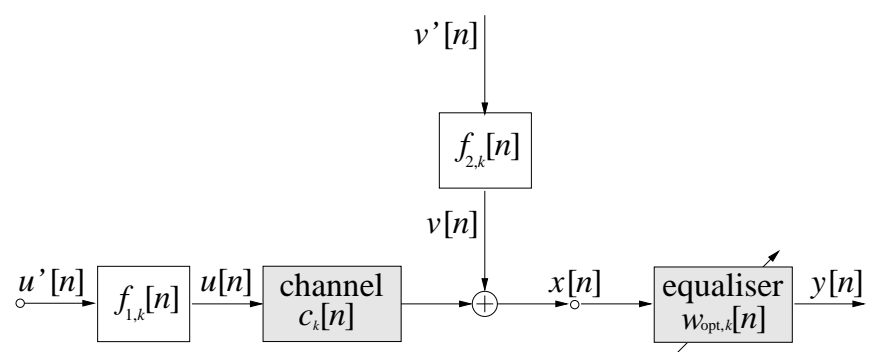

Fig. 4. Block diagram of Wiener filter $w_{\mathrm{opt}, k}[n]$ for $k$ th subband adaptive equaliser.

unit variance $u^{\prime}[n]$ and $v^{\prime}[n]$, respectively. The PDSs of $u[n]$ and $v[n]$ can be written as

$$
P_{u u}\left(e^{j \Omega}\right)=\left|F_{1, k}\left(e^{j \Omega}\right)\right|^{2}
$$

and

$$
P_{v v}\left(e^{j \Omega}\right)=\left|F_{2, k}\left(e^{j \Omega}\right)\right|^{2}
$$

since the PSDs $P_{u^{\prime} u^{\prime}}\left(e^{j \Omega}\right)=P_{v^{\prime} v^{\prime}}\left(e^{j \Omega}\right)=1$, where $f_{1, k}[n] \circ \longrightarrow F_{1, k}\left(e^{j \Omega}\right)$ and $f_{2, k}[n] \circ \longrightarrow F_{2, k}\left(e^{j \Omega}\right)$ denote the Fourier transforms of $f_{1, k}[n]$ and $f_{2, k}[n]$, respectively.

The source model $c_{k}[n]$ defines the decimated unaliased channel component in the $k$ th subband, while $f_{1, k}[n]$ holds the decimated passband and transition bands of the $k$ th analysis filter $h_{k}[n]$ and any potential colouring of $u[n]$. The unaliased signal contribution for the $k$ th subband is defined by the spectral interval of width $2 \pi / N$, centred around the passband of the analysis filter $H_{k}\left(e^{j \Omega}\right) \bullet h_{k}[n]$, and can be represented as

$$
F_{1, k}\left(e^{j \Omega}\right)=\sum_{n=0}^{N-1} H_{k}\left(e^{j(\Omega-2 \pi n) / N}\right) \cdot Q_{k}\left(e^{j(\Omega-2 \pi n) / N}\right)
$$

where $Q_{k}\left(e^{j \Omega}\right)$ is a rectangular window to extract the spectral component of interest. The window is defined as

$Q_{k}\left(e^{j \Omega}\right)= \begin{cases}1, & \text { for } \Omega_{c, k}-\frac{\pi}{N}<\Omega-2 \pi i<\Omega_{c, k}+\frac{\pi}{N} \\ 0, & \text { elsewhere }\end{cases}$

where $i \in \mathbb{N}$ and $\Omega_{c, k}$ is the centre frequency of the passband of $H_{k}\left(e^{j \Omega}\right)$.

Conversely, the source model $f_{2, k}[n]$ in the noise path represents the aliased parts of the $k$ th analysis filter. The alias component can be expressed as

$$
\begin{aligned}
F_{2, k}\left(e^{j \Omega}\right)= & \sum_{n=0}^{N-1} H_{k}\left(e^{j(\Omega-2 \pi n) / N}\right) \\
& \cdot\left(1-Q_{k}\left(e^{j(\Omega-2 \pi n) / N}\right)\right)
\end{aligned}
$$

extracting all the remaining spectral components in the stopband of $H_{k}\left(e^{j \Omega}\right)$. Having defined the quantities in Fig. 4 , we can show that the subband equaliser Wiener solution can be written in terms of (4) and (6) as

$W_{\mathrm{opt}, k}\left(e^{j \Omega}\right)=\frac{\left|\mathrm{F}_{1, k}\left(e^{j \Omega}\right)\right|^{2} \cdot C_{k}^{*}\left(e^{j \Omega}\right)}{\left|F_{1, k}\left(e^{j \Omega}\right)\right|^{2} \cdot\left|C_{k}\left(e^{j \Omega}\right)\right|^{2}+\left|F_{2, k}\left(e^{j \Omega}\right)\right|^{2}}$.

In order to reconstruct an equivalent fullband Wiener filter $W_{\text {opt }}\left(e^{j \Omega}\right)$ from the $K$ subband solutions $W_{\mathrm{opt}, k}\left(e^{j \Omega}\right)$, the adders for the subband errors $e_{k}[m]$ in Fig. 2 are swapped with the linear synthesis filter bank, resulting in the fullband reconstruction model in Fig. 5.

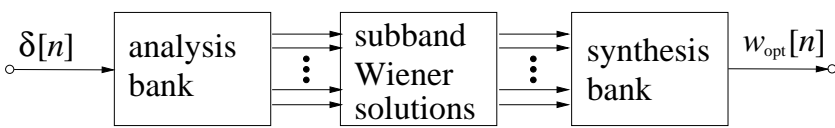

Fig. 5. Reconstruction of equivalent fullband model from subband equaliser system.

The equivalent fullband filter $w_{\text {opt }}[n] \circ-\bullet W_{\text {opt }}\left(e^{j \Omega}\right)$ can be obtained by sending an impulse response $\delta[n]$ through the series of analysis filter bank, subband Wiener solutions and synthesis filter bank. Accordingly, the reconstructed equivalent fullband solution $W\left(e^{j \Omega}\right)$ of any subband equaliser $W_{k}\left(e^{j \Omega}\right)$ can be derived.

\section{Limitation due to Aliasing}

The analytically derived Wiener solution defined in the previous section is compared with simulation results to observe the effect of aliasing. Computer simulations were based on the subband equaliser of Fig. 2 that utilised the LMS algorithm for adaptation. Efficient oversampled GDFT modulated filter banks were employed for subband implementation [11]. The length of equaliser $w[n]$ was selected sufficiently adequate to avoid limitations due to model truncation [12].

We consider two scenarios, whereby no channel noise is added to isolated the limitations imposed by the subband structure. The first scenario consists of a channel impulse response defined by its z-transform $C_{1}(z)=$ $0.292+0.360 z^{-1}+0.756 z^{-2}+0.360 z^{-3}+0.292 z^{-4}$ in combination with a subband equaliser operating in $K=4$ subbands decimated by $N=3$. The results are depicted in Fig. 6. A second scenario is based on a channel $C_{2}(z)=0.192+0.476 z^{-1}+0.688 z^{-2}+$ $0.476 z^{-3}+0.192 z^{-4}$ in conjunction with a subband equaliser in $K=8$ subbands decimated by $N=7$ with characteristics given in Fig. 3. The results are 


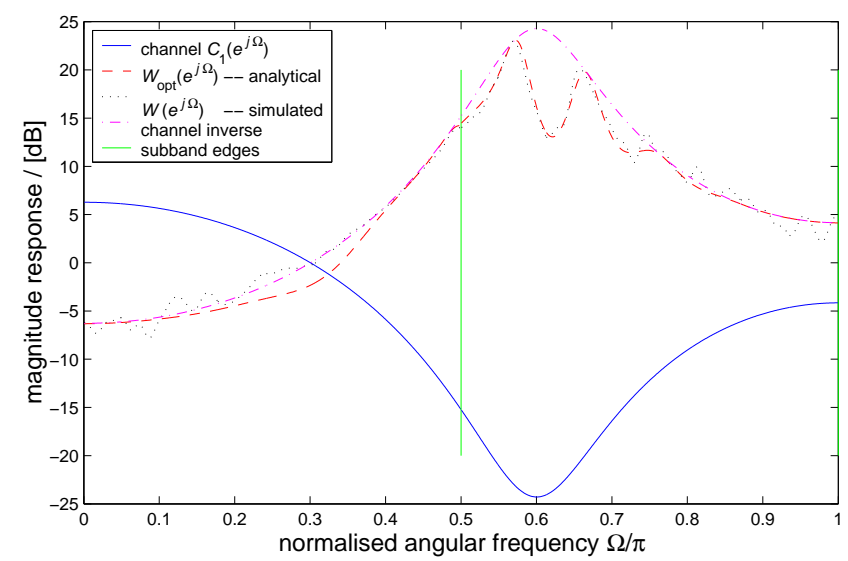

Fig. 6. Comparison between analytical and simulated results of $W\left(e^{j \Omega}\right)$ for channel $C_{1}(z)$, using GDFT modulated filters with $K=4$ number of subband and decimated by a factor of $N=3$.

displayed in Fig. 7 where the subband edges are indicated by the vertical lines.

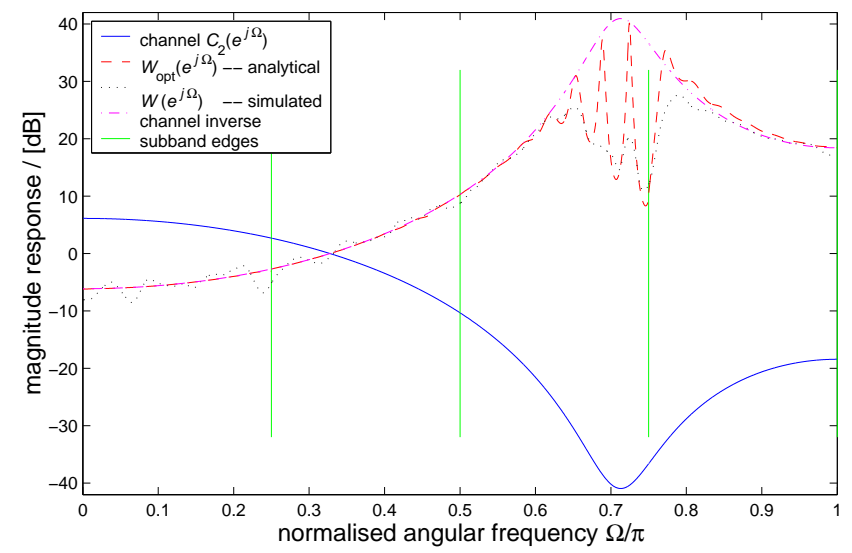

Fig. 7. Comparison between analytical and simulated results of $W\left(e^{j \Omega}\right)$ for channel $C_{2}(z)$, using GDFT modulated filters with $K=8$ number of subband and decimated by a factor of $N=7$.

Fig. 6 shows a good agreement between the analytical calculation - $W_{\text {opt }}\left(e^{j \Omega}\right)$ reconstructed from (7) - and the simulation result - $W\left(e^{j \Omega}\right)$ simulated for channel $C_{1}(z)$ with a clear deviation from the zeroforcing (ZF) solution due to regularisation by aliasing. In the absence of noise, the ZF equalisation result indicated by the channel inverse is the optimum achievable solution. A similar match between the Wiener solution based on the hypothesis on aliasing and the simulated results can be noted for channel $C_{2}(z)$ given in Fig 7 , where again the effect of regularisation due to aliasing can be observed on $W_{\text {opt }}\left(e^{j \Omega}\right)$. Therefore, we have verified the validity of our hypothesis to treat aliasing as channel noise by comparing the simulated answers with the derived the Wiener solution for subband equalisers as discussed in Sec. 3.

\section{Limitation due to Error in Power Complementarity}

Non-perfect filter banks employed in subband systems will limit the steady-state performance of subband adaptive equalisation. If the alias level is suppressed below the channel spectral dynamic, an illustration of error in power complementarity limitation on achievable MMSE solution is shown in Fig. 8 (top) where $W_{\text {opt }}\left(e^{j \Omega}\right)$ failed to compensate the distortion caused by channel $C_{1}(z)$. For comparison the channel inverse is also plotted to indicate the optimum solution for the noiseless channel. There are obvious notches in the equaliser $W_{\text {opt }}\left(e^{j \Omega}\right)$ at the band edges caused by the guard bands of the non-perfect filter banks. Note that simulation result $W\left(e^{j \Omega}\right)$ compares favourably with the analytical derivation $W_{\text {opt }}\left(e^{j \Omega}\right)$. The spectral characteristic of the employed non-PR GDFT modulated filter banks is depicted in Fig. 8 (bottom).

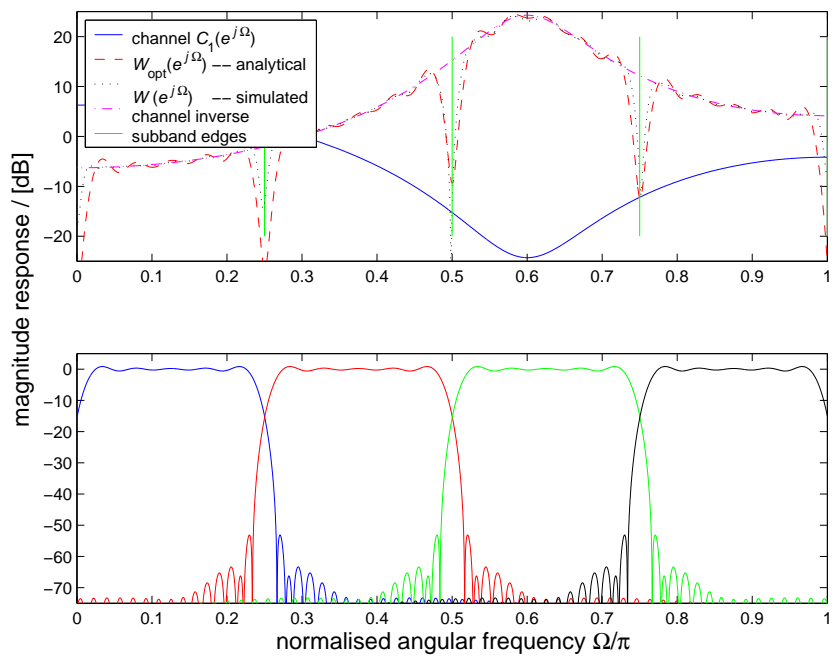

Fig. 8. (top) Comparison between analytical and simulated results of $W\left(e^{j \Omega}\right)$ for channel $C_{1}(z)$, using non-PR GDFT modulated filter banks. (bottom) Magnitude response and arrangement of bandpass filters with $K=8$ number of subband and decimated by a factor of $N=7$, only showing half the bands covering the spectrum $[0 ; \pi]$. 


\section{Discussion}

The performance of subband adaptive equalisers is limited by aliasing as well as errors in power complementarity. Specifically, we have shown the limitations on the optimum MMSE solution of subband system as presented in previous sections.

We established a hypothesis that the subband equaliser's performance is limited by aliasing in a way that noise would degrade its performance and derived the Wiener solution for such system. Results from both analytical derivation and computer simulation have verified this hypothesis. Aliasing can be considered equivalent to channel noise, which permits to quantify its effect on the optimum solution. In absence of channel noise, the alias level should be lower than the channel spectral dynamic. Nevertheless, in a real application, the equaliser would be prone to true channel noise and consequently the regularisation term would be influenced by both aliasing and noise. Therefore, as long as the alias level of the subband equaliser remains below the injected channel noise, no MMSE limitations of the subband equaliser with respect to a fullband system are incurred. Thus, when designing a subband equaliser it is important to select the filter banks sufficient with respect to the channel SNR. Vice versa, the filter design only has to be good enough and therefore can be traded-off for, for example, low delay properties.

Limitations on the steady state performance are also imposed by the use of non-PR filter banks. For the sake of completion, the effect of errors in power complementarity on the MMSE equaliser has been illustrated in Sec. 5 where the results from both analytical derivation and simulation were plotted. Hence, the subband adaptive equaliser must employ PR or near-PR filter banks to ensure successful equalisation.

\section{Conclusions}

In this paper, we have demonstrated that the optimum subband adaptive equaliser is limited by aliasing and errors in power complementarity. The former is due to decimation, which, as claimed in our hypothesis, can be assumed to be equivalent to channel noise. Simulation results confirmed the validity of this hypothesis by deriving the Wiener solution with a regularisation by aliasing. The use of PR filter banks is mandatory whilst the effect of aliasing should be minimised below the channel SNR in order to guarantee satisfactory performance of the subband system. This finding provides an important guideline for subband equaliser design with respect to achieving sufficient performance in a real situation.

\section{References}

[1] S. U. H. Qureshi, "Adaptive Equalization", Proceeding of the IEEE, vol. 73, pp. 1349-1387, Sept. 1985.

[2] H. Mohamad, S. Weiss, M. Rupp, and L. Hanzo, "Fast Adaptation of Fractionally Spaced Equalisers," IEE Electronics Letters, vol. 38, no. 2, pp. 9698, Jan. 2002.

[3] H. Mohamad, S. Weiss, M. Rupp, and L. Hanzo, "A Performance Comparison of Fullband and Different Subband Adaptive Equalisers," in Proceedings of the 11th IEEE Signal Processing Workshop on Statistical Signal Processing, pp. 567-570, Aug. 2001.

[4] W. Kellermann, "Analysis and Design of Multirate Systems for Cancellation of Acoustical Echoes," in Proc. IEEE ICASSP, vol. 5, pp. 2570-2573, New York, 1988.

[5] A. Gilloire and M. Vetterli, "Adaptive Filtering in Subbands with Critical Sampling: Analysis, Experiments and Applications to Acoustic Echo Cancellation," IEEE Transactions on Signal Processing, vol. SP-40, no. 8, pp. 1862-1875, August 1992.

[6] S. Weiss, A. Stenger, R. W. Stewart, and R. Rabenstein, "Steady-State Performance Limitations of Subband Adaptive Filters", IEEE Transaction on Signal Processing, vol. 49, no. 9, pp. 1982-1991, 2001.

[7] M. Harteneck, S. Weiss, and R. W. Stewart, "Design of Near Perfect Reconstruction Oversampled Filter Banks for Subband Adaptive Filters," IEEE Transactions on Circuits and Systems II, vol. 46, no. 8, pp. 1081-1086, August 1999.

[8] P. P. Vaidyanathan, Multirate Systems and Filter Banks, Prentice Hall, 1993.

[9] B. Girod, R. Rabenstein, and A. Stenger, Signals and Systems, J. Wiley \& Sons, Chichester, 2001.

[10] R. A. Casas, F. Lopez de Victoria, I. Fijalkow, P. Schniter, T. J. Endrea, and C. R. Johnson, Jr. "On MMSE fractionally-spaced equalizer design," in Proceedings of 13th International Conference on Signal Processing, vol. 1, pp. 395-398, July 1997.

[11] S. Weiss, and R. W. Stewart, " Fast Implementation of Oversampled Modulated Filter Banks", Electronics Letters, vol. 36, no. 17, pp. 1502 -1503, Aug. 2000.

[12] S. Haykin, Adaptive Filter Theory, Prentice Hall, 3rd ed., 1996. 